\title{
Erratum to: The Dirichlet problem associated to the relativistic heat equation
}

\author{
Fuensanta Andreu ${ }^{1}$. Vicent Caselles ${ }^{2}$. \\ Jose M. Mazón ${ }^{1}$. Salvador Moll ${ }^{1}$
}

Published online: 16 May 2015

(C) Springer-Verlag Berlin Heidelberg 2015

\section{Erratum to: Math. Ann. (2010) 347:135-199 DOI 10.1007/s00208-009-0428-3}

In the original article we proved existence and uniqueness of entropy solutions for the nonhomogeneous Dirichlet problem associated to the relativistic heat equation. In the proof of uniqueness of both elliptic and parabolic entropy solutions (Theorems 2 and 3 , respectively) we used that $\operatorname{sign}_{0}^{+}(u-\bar{u}) u \in B V(\Omega)$ for two entropy solutions $u, \bar{u}$. However, we did not prove this but we wrongly stated that this was true for any given functions $u, \bar{u} \in T B V(\Omega)$, which is not the case, in general.

The expression $\operatorname{sign}_{0}^{+}(u-\bar{u}) u$ appears in the process of applying the doubling variables method, known as Kruzhkov's method, if one lets the parameter $\varepsilon \rightarrow 0^{+}$, which serves to approximate the sign function, before joining the space variables. In this note, we correct the aforementioned mistake by joining first the space variables and at the very end, letting $\varepsilon \rightarrow 0^{+}$.

The online version of the original article can be found under doi:10.1007/s00208-009-0428-3.

Jose M. Mazón

Jose.M.Mazon@uv.es; mazon@uv.es

Fuensanta Andreu

f.andreu@uv.es

Vicent Caselles

vicent.caselles@upf.edu

Salvador Moll

j.salvador.moll@uv.es

1 Departament d'Anàlisi Matemàtica, Universitat de València, Burjassot, Spain

2 Departament de Tecnologia, Universitat Pompeu Fabra, Barcelona, Spain 
We assume here all notations in the original article. Moreover, due to the fact that $u(t, x)$ is a solution to $u_{t}=v \operatorname{div}\left(\frac{u \nabla u}{\sqrt{u^{2}+\frac{v^{2}}{c^{2}}|\nabla u|^{2}}}\right)$ if and only if $v(t, x)=u\left(\frac{v t}{c^{2}}, \frac{v}{c} x\right)$ is a solution to $v_{t}=\operatorname{div}(\mathbf{a}(v, D v))$ with $\mathbf{a}(z, \xi):=\frac{z \xi}{\sqrt{z^{2}+|\xi|^{2}}}$, for the sake of simplicity, we assume that $v=c=1$.

In order to be able to join first the space variables in Kruzkov's method, we need first some auxiliary results.

Lemma 1 Let $U \subset \mathbb{R}^{k}$ be an open set. Let $\mu \in \mathcal{M}(U)$ be such that $|\mu|(U)<+\infty$. Then, given an uncountable collection of $\mu$-measurable disjoint subsets $\left\{B_{\varepsilon}\right\}$ of $U$ it holds

$$
\#\left\{\varepsilon:|\mu|\left(B_{\varepsilon}\right)>0\right\} \leq \aleph_{0} .
$$

Proof By contradiction, suppose that $A:=\left\{\varepsilon:|\mu|\left(B_{\varepsilon}\right)>0\right\}$ is uncountable. Then, there is $n \in \mathbb{N}$ such that the set $A_{n}:=\left\{\varepsilon:|\mu|\left(B_{\varepsilon}\right)>\frac{1}{n}\right\} \subseteq A$ is uncountable (otherwise one could write $A=\cup_{n=1}^{\infty} A_{n}$ and $A$ would be countable). This implies that there exists a sequence $\left\{\varepsilon_{k}\right\}_{k=0}^{\infty} \subset A_{n}$. By $\sigma$-additivity of $\mu$ we would have a contradiction since

$$
+\infty=\sum_{k=0}^{\infty}|\mu|\left(B_{\varepsilon_{k}}\right)=|\mu|\left(\bigcup_{k=0}^{\infty} B_{\varepsilon_{k}}\right) \leq|\mu|(U)<+\infty .
$$

Let $w, \bar{w} \in L^{1}(\Omega), \psi \in \mathcal{D}(\Omega)$ and $F \in W^{1, \infty}(\mathbb{R})$. Given $x \in \Omega \backslash S_{\bar{w}}$, we define $f_{x}: \Omega \rightarrow \mathbb{R}$ as

$$
f_{x}(y):=\psi\left(\frac{y+x}{2}\right) \chi_{\{0<w(y)-\bar{w}(x)<\varepsilon\}}(F(w)(y)-F(\bar{w})(x)) .
$$

Lemma 2 Let $\rho_{n}$ be a sequence of classical mollifiers in $\Omega$ and let $w, \bar{w} \in B V(\Omega)$. Then, if $\left|D^{c} \bar{w}\right|(\{\tilde{w}-\tilde{\bar{w}}=\varepsilon\})=0$ it holds

$$
\int_{\Omega}\left(f_{x} * \rho_{n}\right)(x) d\left|D^{c} \bar{w}\right| \stackrel{n \rightarrow \infty}{\rightarrow} \int_{\Omega} f_{x}(x) d\left|D^{c} \bar{w}\right| .
$$

Proof Since $J_{w}$ is $\mathcal{H}^{N-1}$ rectifiable, then $J_{w}$ is $\sigma$-finite with respect to the Haussdorff measure $\mathcal{H}^{N-1}$. Then, by [1, Proposition 3.92], it follows that $\left|D^{c} \bar{w}\right|\left(S_{w}\right)=$ $\left|D^{c} \bar{w}\right|\left(J_{w}\right)=0$. Hence,

$$
\int_{\Omega}\left(f_{x} * \rho_{n}\right)(x) d\left|D^{c} \bar{w}\right|=\int_{\Omega \backslash\left(S_{\bar{w}} \cup S_{w}\right)}\left(f_{x} * \rho_{n}\right)(x) d\left|D^{c}(\bar{w})\right| .
$$

Since $f_{x} \in L^{1}(\Omega)$, we have that $\left(f_{x} * \rho_{n}\right)(y) \rightarrow f_{x}(y)$ for all $y \in \Omega \backslash S_{f_{x}}$. On the other hand, if $x \in \Omega \backslash\left(S_{w} \cup S_{\bar{w}}\right)$, then 
$x \in \Omega \backslash S_{f_{x}} \Longleftrightarrow x \in \Omega \backslash S_{\chi_{\{0<w(\cdot)-\bar{w}(x)<\varepsilon\}}}=\Omega \backslash \partial^{*}\{0<w(\cdot)-\bar{w}(x)<\varepsilon\}$.

Now, $\left.\partial^{*}(\{0<w(\cdot)-\bar{w}(x)<\varepsilon\}) \subset\{w(\cdot)=\bar{w}(x)\} \cup\{w(\cdot)-\bar{w}(x)=\varepsilon\}\right)$. Observe also that $x \in\{w(\cdot)-\bar{w}(x)=\varepsilon\}$ if and only if $x \in\{w-\bar{w}=\varepsilon\}$.

In case $w(x)=\bar{w}(x)$,

$$
\begin{aligned}
\left|\left(f_{x} * \rho_{n}\right)(x)\right| & \leq \int_{\Omega} \rho_{n}(x-y)\left|f_{x}(y)\right| d y \\
& \leq\|\nabla F\|_{\infty} \int_{\Omega} \rho_{n}(x-y) \psi\left(\frac{x+y}{2}\right)|w(y)-\bar{w}(x)| d y \rightarrow 0=f_{x}(x)
\end{aligned}
$$

Then, if $x \in \Omega \backslash\left(S_{w} \cup S_{\bar{w}} \cup\{\tilde{w}-\tilde{\bar{w}}=\varepsilon\}\right)$, we obtain that $f_{x} * \rho_{n}(x) \rightarrow f_{x}(x)$ pointwise. Moreover, since $\left\|f_{x} * \rho_{n}\right\|_{\infty} \leq\left\|f_{x}\right\|_{\infty}$, we can apply the dominated convergence Theorem to obtain that

$$
\int_{\Omega \backslash\left(S_{w} \cup S_{\bar{w}} \cup\{\tilde{w}-\tilde{\bar{w}}=\varepsilon\}\right)}\left(f_{x} * \rho_{n}\right)(x) d\left|D^{c} \bar{w}\right| \stackrel{n \rightarrow \infty}{\rightarrow} \int_{\Omega \backslash\left(S_{w} \cup S_{\bar{w}} \cup\{\tilde{w}-\tilde{\bar{w}}=\varepsilon\}\right)} f_{x}(x) d\left|D^{c} \bar{w}\right|
$$

Therefore,

$$
\int_{\Omega}\left(f_{x} * \rho_{n}\right)(x) d\left|D^{c} \bar{w}\right| \stackrel{n \rightarrow \infty}{\rightarrow} \int_{\Omega} f_{x}(x) d\left|D^{c} \bar{w}\right|
$$

Let $w, \bar{w} \in B V(\Omega)$ and $\psi \in \mathcal{D}(\Omega)$. Given $x \in \Omega \backslash\left(S_{\bar{w}} \cup S_{\nabla \bar{w}}\right)$, we define $g_{x}$ : $\Omega \rightarrow \mathbb{R}$ as

$$
g_{x}(y):=\psi\left(\frac{y+x}{2}\right) \chi_{\{0<w(y)-\bar{w}(x)<\varepsilon\}}(w(y)-\bar{w}(x))\left|\nabla w(y)-\nabla_{x} \bar{w}(x)\right| .
$$

Lemma 3 Let $\rho_{n}$ be a sequence of classical mollifiers in $\Omega$. If $\mathcal{L}^{N}(\{w=\bar{w}+\varepsilon\})=0$, then

$$
\int_{\Omega}\left(g_{x} * \rho_{n}\right)(x) d x \stackrel{n \rightarrow \infty}{\rightarrow} \int_{\Omega} g_{x}(x) d x
$$

Proof Let us denote by $\tilde{\Omega}:=\Omega \backslash\left(S_{w} \cup S_{\nabla w}\right)$. Then,

$$
\begin{aligned}
\int_{\Omega}\left(g_{x} * \rho_{n}\right)(x) d x & =\int_{\tilde{\Omega}}\left(g_{x} * \rho_{n}\right)(x) d x \\
& =\int_{\tilde{\Omega} \cap\{w=\bar{w}\}}\left(g_{x} * \rho_{n}\right)(x) d x+\int_{\tilde{\Omega} \backslash\{w=\bar{w}\}}\left(g_{x} * \rho_{n}\right)(x) d x .
\end{aligned}
$$


For the first term,

$$
\begin{aligned}
\left|\int_{\tilde{\Omega} \cap\{w=\bar{w}\}}\left(g_{x} * \rho_{n}\right)(x) d x\right| & \leq C \int_{\tilde{\Omega} \cap\{w=\bar{w}\}} n^{N} \int_{\Omega \cap B_{\frac{1}{n}}(x)}\left|\nabla w(y)-\nabla_{x} \bar{w}(x)\right| d y d x \\
& \rightarrow \frac{C}{\omega_{N}} \int_{\tilde{\Omega} \cap\{w=\bar{w}\}}|\nabla w(x)-\nabla \bar{w}(x)| d x \stackrel{[1, \text { Remark 3.93] }}{=} 0
\end{aligned}
$$

For the second term, we split $\tilde{\Omega} \backslash\{w=\bar{w}\}$ into two Borel sets: $\tilde{\Omega}_{1}:=\tilde{\Omega} \backslash(\{w=$ $\bar{w}\} \cup\{w=\bar{w}+\varepsilon\}), \tilde{\Omega}_{2}:=\tilde{\Omega} \cap\{w=\bar{w}+\varepsilon\}$. Observe that in $\tilde{\Omega}_{2}$ both the integral and its limit are equal to 0 and we do not have to prove anything. In $\tilde{\Omega}_{1}$ instead, all points are Lebesgue points of $g_{x}$. Therefore, applying dominated convergence Theorem (note that $\left.\left\|g_{x} * \rho_{n}\right\|_{1} \leq\left\|g_{x}\right\|_{1}<+\infty\right)$ we obtain the result.

With an easy adaptation of the proofs of the preceding results, one can obtain their time dependent counterparts.

Lemma 4 Let $\rho_{n}, \rho_{m}$ be sequences of classical mollifiers in $\Omega$ and $(0, T)$, respectively, $w, \bar{w} \in L_{l o c, w}^{1}(0, T ; B V(\Omega)), \psi \in \mathcal{D}(\Omega)$ and $f_{x}(s, t)(y):=\rho_{m}(s-t) \psi\left(\frac{y+x}{2}\right) \chi_{\{0<w(s, y)-\bar{w}(t, x)<\varepsilon\}}(F(w)(s, y)-F(\bar{w})(t, x))$.

Then, if $\left.\int_{(0, T)^{2}}\left|D^{c} \bar{w}(s)\right|(\{\widetilde{w(s)}-\widetilde{\bar{w}(t)}=\varepsilon\})\right) d s d t=0$ it holds

$$
\begin{gathered}
\int_{(0, T)^{2}} \int_{\Omega}\left(f_{x}(s, t) * \rho_{n}\right)(x) d\left|D^{c} \bar{w}(s)\right| d s d t \\
\stackrel{n \rightarrow \infty}{\rightarrow} \int_{(0, T)^{2}} \int_{\Omega} f_{x}(s, t)(x) d\left|D^{c} \bar{w}(s)\right| d s d t .
\end{gathered}
$$

Lemma 5 Let $\rho_{n} \rho_{m}$ be sequences of classical mollifiers in $\Omega$ and $(0, T)$, respectively, $w, \bar{w} \in L_{l o c, w}^{1}(0, T ; B V(\Omega)), \psi \in \mathcal{D}(\Omega)$ and

$$
\begin{aligned}
g_{x}(s, t)(y):= & \rho_{m}(s-t) \psi\left(\frac{y+x}{2}\right) \chi_{\{0<w(s, y)-\bar{w}(t, x)<\varepsilon\}} \\
& \times(w(s, y)-\bar{w}(t, x))\left|\nabla w(s, y)-\nabla_{x} \bar{w}(t, x)\right| .
\end{aligned}
$$

If $\mathcal{L}^{N+2}(\{w(s)=\bar{w}(t)+\varepsilon\})=0$, then

$$
\int_{(0, T)^{2}} \int_{\Omega}\left(g_{x}(s, t) * \rho_{n}\right)(x) d x d s d t \stackrel{n \rightarrow \infty}{\rightarrow} \int_{(0, T)^{2}} \int_{\Omega} g_{x}(s, t)(x) d x d s d t .
$$

We have now all the ingredients for the proofs of uniqueness. Note that in the proof of Theorem 2 of the orginial article the mistake takes place in passing from (41) up to (42) while in the proof of Theorem 3 of the original article from (124) up to (130). Since both of the proofs are similar, we will give the whole proof for the elliptic case 
(up to (42) in the original article) and we will only sketch the one for the parabolic case.

In the statement of Theorem 2 of the original article, there was also a mistake. The correct statement is the following one.

Theorem 1 Given $0 \leq g \in L^{\infty}(\Omega), v, \bar{v} \in L^{1}(\Omega), v \geq 0, \bar{v} \geq 0$, let $u, \bar{u}$ be two bounded entropy solutions of the problems

$$
\begin{cases}u-\operatorname{div} \mathbf{a}(u, D u)=v & \text { in } \Omega \\ u=g & \text { on } \partial \Omega\end{cases}
$$

and

$$
\begin{cases}\bar{u}-\operatorname{div} \mathbf{a}(\bar{u}, D \bar{u})=\bar{v} & \text { in } \Omega \\ \bar{u}=g & \text { on } \partial \Omega\end{cases}
$$

respectively. Then,

$$
\int_{\Omega}(u-\bar{u})^{+} \leq \int_{\Omega}(v-\bar{v})^{+} .
$$

Proof For $a>0$, we denote by $T_{a}^{\infty}$ the truncature function

$$
T_{a}^{\infty}(s):= \begin{cases}a & \text { if } \quad s \leq a \\ s & \text { if } s \geq a .\end{cases}
$$

Let $b>a>0$. We note that by Lemma 1 , we can choose $\varepsilon \rightarrow 0$ such that $\varepsilon<\frac{a}{2}$,

$$
\mathcal{L}^{N}\left\{T_{a}^{\infty} \tilde{u}-T_{\frac{a}{2}}^{\infty} \tilde{\bar{u}}=\varepsilon\right\}=0
$$

and $\left(\left|D^{c} T_{a}^{\infty} u\right|+\left|D^{c} T_{\frac{a}{2}}^{\infty} \bar{u}\right|\right)\left\{T_{a}^{\infty} \tilde{u}-T_{\frac{a}{2}}^{\infty} \tilde{\bar{u}}=\varepsilon\right\}=0$. Observe now that

$$
\left\{T_{a}^{\infty} \tilde{u}-T_{\frac{a}{2}}^{\infty} \tilde{\bar{u}}=\varepsilon\right\} \cap\{\tilde{u}>a\}=\left\{T_{a}^{\infty} \tilde{u}-T_{a-\varepsilon}^{\infty} \tilde{\bar{u}}=\varepsilon\right\} \cap\{\tilde{u}>a\},
$$

which implies that $\left|D^{c} T_{a}^{\infty} u\right|\left\{T_{a}^{\infty} \tilde{u}-T_{a-\varepsilon}^{\infty} \tilde{\bar{u}}=\varepsilon\right\}=0$. On the other hand,

$$
\begin{aligned}
\left|D^{c} T_{a-\varepsilon}^{\infty} \bar{u}\right|\left\{T_{a}^{\infty} \tilde{u}-T_{a-\varepsilon}^{\infty} \tilde{\bar{u}}=\varepsilon\right\} & =\left|D^{c} T_{a-\varepsilon}^{\infty} \bar{u}\right|\left\{T_{a}^{\infty} \tilde{u}-T_{\frac{a}{2}}^{\infty} \tilde{\bar{u}}=\varepsilon\right\} \\
& \leq\left|D^{c} T_{\frac{a}{2}}^{\infty} \bar{u}\right|\left\{T_{a}^{\infty} \tilde{u}-T_{\frac{a}{2}}^{\infty} \frac{\tilde{u}}{=}=\varepsilon\right\}=0
\end{aligned}
$$

Therefore,

$$
\left(\left|D^{c} T_{a}^{\infty} u\right|+\left|D^{c} T_{a-\varepsilon}^{\infty} \bar{u}\right|\right)\left\{T_{a}^{\infty} \tilde{u}-T_{a-\varepsilon}^{\infty} \tilde{\bar{u}}=\varepsilon\right\}=0 .
$$

We consider $T(r):=T_{a, b}(r)-a, S_{\varepsilon, l}(r):=T_{\varepsilon}(r-l)^{+}=T_{l, l+\varepsilon}(r)-l \in \mathcal{P}^{+}$and $S_{\varepsilon}^{l}(r):=T_{\varepsilon}(r-l)^{-}+\varepsilon=T_{l-\varepsilon, l}(r)+\varepsilon-l \in \mathcal{P}^{+}$, where $l \geq 0$. Let us denote 


$$
J_{T, \varepsilon, l}^{+}(r)=\int_{l}^{r} T(s) T_{\varepsilon}(s-l)^{+} d s, J_{T, \varepsilon, l}^{-}(r)=\int_{l}^{r} T(s) T_{\varepsilon}(s-l)^{-} d s .
$$

Given $0 \leq g \in L^{\infty}(\Omega), v, \bar{v} \in L^{1}(\Omega), v \geq 0, \bar{v} \geq 0$, let $u, \bar{u}$ be bounded entropy solutions of the problems (1) and (2), respectively. Let $\rho_{n}$ be a sequence of classical mollifiers in $\Omega, 0 \leq \psi \in \mathcal{D}(\Omega)$ and $b>a>2 \varepsilon>0$. We write $\xi_{n}(x, y)$ $=\rho_{n}(x-y) \psi\left(\frac{x+y}{2}\right)$.

If we denote $\mathbf{z}(y)=\mathbf{a}(u(y), \nabla u(y))$ and $\overline{\mathbf{z}}(x)=\mathbf{a}(\bar{u}(x), \nabla \bar{u}(x))$, we have

$$
u-\operatorname{div}(\mathbf{z})=v \quad \text { and } \quad \bar{u}-\operatorname{div}(\overline{\mathbf{z}})=\bar{v} \quad \text { in } \quad \mathcal{D}^{\prime}(\Omega) .
$$

Multiplying Eq. (1) by $T(u) S_{\varepsilon, \bar{u}}(u) \xi_{n}$ and (2) by $T(\bar{u}) S_{\varepsilon}^{u}(\bar{u}) \xi_{n}$ and integrating by parts, integrating again in $x$ and $y$ respectively and adding both equations, we obtain

$$
\begin{aligned}
& \int_{\Omega} \int_{\Omega}(u T(u)-\bar{u} T(\bar{u})) T_{\varepsilon}(u-\bar{u})^{+} \xi_{n} d x d y+\varepsilon \int_{\Omega} \int_{\Omega}(\bar{u}-\bar{v}) T(\bar{u}) \xi_{n} d x d y \\
& \quad+\int_{\Omega}\left(\int_{\Omega} \xi_{n}\left(\mathbf{z}, D_{y}\left(T(u) S_{\varepsilon, \bar{u}}(u)\right)\right) d x+\int_{\Omega \times \Omega} T(u) S_{\varepsilon, \bar{u}}(u) \mathbf{z} \cdot \nabla_{y} \xi_{n} d y d x\right. \\
& \quad+\int_{\Omega}\left(\int_{\Omega} \xi_{n}\left(\overline{\mathbf{z}}, D_{x}\left(T(\bar{u}) S_{\varepsilon}^{u}(\bar{u})\right)\right)\right) d y+\int_{\Omega \times \Omega} T(\bar{u}) S_{\varepsilon}^{u}(\bar{u}) \overline{\mathbf{z}} \cdot \nabla_{x} \xi_{n} d x d y \\
& =\int_{\Omega} \int_{\Omega}(v T(u)-\bar{v} T(\bar{u})) T_{\varepsilon}(u-\bar{u})^{+} \xi_{n} d x d y .
\end{aligned}
$$

Let $I_{1}, I_{2}$ be, respectively, the first term and the rest of the terms at the left hand side of the above identity, and let $I_{3}$ be the right hand side term.

Now, since $\bar{u}-\bar{v}=\operatorname{div} \overline{\mathbf{z}}$ and $\nabla_{y} \xi_{n}(x, y)+\nabla_{x} \xi_{n}(x, y)=\rho_{n} \nabla \psi$, we have

$$
\begin{aligned}
& I_{2}=\varepsilon \int_{\Omega} \int_{\Omega} T(\bar{u})\left(\operatorname{div}(\overline{\mathbf{z}}) \xi_{n}+\overline{\mathbf{z}} \cdot \nabla_{x} \xi_{n}\right) d x d y \\
& +\int_{\Omega}\left(\int_{\Omega} \xi_{n}\left(\mathbf{z}, D_{y}\left(T(u) S_{\varepsilon, \bar{u}(x)}(u)\right)\right)\right) d x+\int_{\Omega \times \Omega} T(\bar{u}) T_{\varepsilon}(u-\bar{u})^{+} \overline{\mathbf{z}} \cdot \nabla_{y} \xi_{n} d x d y \\
& +\int_{\Omega}\left(\int_{\Omega} \xi_{n}\left(\overline{\mathbf{z}}, D_{x}\left(T(\bar{u}) S_{\varepsilon}^{u(y)}(\bar{u})\right)\right)\right) d y-\int_{\Omega \times \Omega} T(u) T_{\varepsilon}(u-\bar{u})^{+} \mathbf{z} \cdot \nabla_{x} \xi_{n} d y d x \\
& +\int_{\Omega \times \Omega} \rho_{n} T_{\varepsilon}(u-\bar{u})^{+}(T(u) \mathbf{z}-T(\bar{u}) \overline{\mathbf{z}}) \cdot \nabla \psi=\varepsilon \int_{\Omega} \int_{\Omega} T(\bar{u}) \operatorname{div}\left(\overline{\mathbf{z}} \xi_{n}\right) d x d y \\
& +\int_{\Omega}\left(\int_{\Omega} \xi_{n}\left(\mathbf{z}, D_{y}\left(T(u) S_{\varepsilon, \bar{u}(x)}(u)\right)\right)\right) d x-\int_{\Omega \times \Omega} \xi_{n} \overline{\mathbf{z}} \cdot D_{y}\left(T(\bar{u}) T_{\varepsilon}(u-\bar{u})^{+}\right) d x d y \\
& \left.+\int_{\Omega}\left(\int_{\Omega} \xi_{n}\left(\overline{\mathbf{z}}, D_{x}\left(T(\bar{u}) S_{\varepsilon}^{u(y)}(\bar{u})\right)\right)\right) d y+\int_{\Omega \times \Omega} \xi_{n} \mathbf{z} \cdot D_{x}\left(T(u) T_{\varepsilon}(u-\bar{u})^{+}\right)\right) d y d x \\
& +\int_{\Omega \times \Omega} \rho_{n} T_{\varepsilon}(u-\bar{u})^{+}(T(u) \mathbf{z}-T(\bar{u}) \overline{\mathbf{z}}) \cdot \nabla \psi=-\varepsilon \int_{\Omega} \int_{\Omega} \xi_{n}(\overline{\mathbf{z}}, D T(\bar{u})) d y \\
& +\int_{\Omega}\left(\int_{\Omega} \xi_{n}\left(\mathbf{z}, D_{y} J_{T^{\prime} S_{\varepsilon, \bar{u}(x)}}(u)\right)\right) d x+\int_{\Omega}\left(\int_{\Omega} \xi_{n}\left(\overline{\mathbf{z}}, D_{x} J_{T^{\prime} S_{\varepsilon}^{u(y)}}(\bar{u})\right)\right) d y
\end{aligned}
$$




$$
\begin{aligned}
& +\int_{\Omega}\left(\int_{\Omega} \xi_{n}\left(\mathbf{z}, D_{y} J_{T S_{\varepsilon, \bar{u}(x)}^{\prime}}(u)\right)\right) d x-\int_{\Omega} T(\bar{u})\left(\int_{\Omega} \xi_{n} \overline{\mathbf{z}} \cdot D_{y} T_{\varepsilon}(u-\bar{u})^{+}\right) d x \\
& +\int_{\Omega}\left(\int _ { \Omega } \xi _ { n } \left(\overline{\mathbf{z}}, D_{x} J_{\left.\left.T S_{\varepsilon}^{u(y) \prime}(\bar{u})\right)\right)} d y+\int_{\Omega} T(u(y))\left(\int_{\Omega} \xi_{n} \mathbf{z} \cdot D_{x} T_{\varepsilon}(u-\bar{u})^{+}\right) d y\right.\right. \\
& +\int_{\Omega \times \Omega} \rho_{n} T_{\varepsilon}(u-\bar{u})^{+}(T(u) \mathbf{z}-T(\bar{u}) \overline{\mathbf{z}}) \cdot \nabla \psi=I_{2}^{1}+I_{2}^{2},
\end{aligned}
$$

where $I_{2}^{1}$ denotes the sum of the first three terms and the last one while $I_{2}^{2}$ denotes the sum from the fourth to the seventh terms.

Let us consider the second and third terms in $I_{2}^{1}$. Since by Definition 1 in the original article,

$$
\begin{aligned}
h_{S_{\varepsilon, \bar{u}(x)}}(u, D T(u)) & \leq\left(\mathbf{z}, D_{y} J_{T^{\prime} S_{\varepsilon, \bar{u}(x)}}(u)\right) \text { and } \\
h_{S_{\varepsilon}^{u(y)}}(\bar{u}, D T(\bar{u})) & \leq\left(\overline{\mathbf{z}}, D_{x} J_{T^{\prime} S_{\varepsilon}^{u(y)}}(\bar{u})\right)
\end{aligned}
$$

as measures in $\Omega$ (see Eq. (30) in the original article for the Definitions of the measures $h_{S}$ ), we have

$$
\begin{aligned}
& \int_{\Omega}\left(\int_{\Omega} \xi_{n}\left(\mathbf{z}, D_{y} J_{\left.T^{\prime} S_{\varepsilon, \bar{u}(x)}\right)}(u)\right)\right) d x \geq 0 \text { and } \\
& \int_{\Omega}\left(\int_{\Omega} \xi_{n}\left(\overline{\mathbf{z}}, D_{x} J_{T^{\prime} S_{\varepsilon}^{u(y)}}(\bar{u})\right)\right) d y \geq 0 .
\end{aligned}
$$

Hence,

$$
I_{2}^{1} \geq \varepsilon \int_{\Omega} \int_{\Omega} \xi_{n}(\overline{\mathbf{z}}, D T(\bar{u})) d y+\int_{\Omega^{2}} \rho_{n} T_{\varepsilon}(u-\bar{u})^{+}(T(u) \mathbf{z}-T(\bar{u}) \overline{\mathbf{z}}) \cdot \nabla \psi
$$

We split $I_{2}^{2}$ into $I_{2}^{2}=I_{2}^{2}(a c)+I_{2}^{2}(s)$, where $I_{2}^{2}(a c)$ contains the absolutely continuous parts of the integrands in $I_{2}^{2}$ and $I_{2}^{2}(s)$ contains their singular parts. Now,

$$
\begin{aligned}
I_{2}^{2}(a c)= & \int_{\Omega} \int_{\Omega} \xi_{n} T(u) \mathbf{z} \cdot \nabla_{y} T_{\varepsilon}(u-\bar{u})^{+} d y d x \\
& -\int_{\Omega} \int_{\Omega} \xi_{n} T(\bar{u}) \overline{\mathbf{z}} \cdot \nabla_{y} T_{\varepsilon}(u-\bar{u})^{+} d y d x \\
& -\int_{\Omega} \int_{\Omega} \xi_{n} T(\bar{u}) \overline{\mathbf{z}} \cdot \nabla_{x} T_{\varepsilon}(u-\bar{u})^{+} d x d y \\
& +\int_{\Omega} \int_{\Omega} \xi_{n} T(u) \mathbf{z} \cdot \nabla_{x} T_{\varepsilon}(u-\bar{u})^{+} d x d y \\
= & \int_{\Omega} \int_{\Omega} \xi_{n}(\mathbf{z} T(u)-\overline{\mathbf{z}} T(\bar{u}))\left(\nabla_{y} T_{\varepsilon}(u-\bar{u})^{+}+\nabla_{x} T_{\varepsilon}(u-\bar{u})^{+}\right) d x d y \\
= & \int_{\Omega} \int_{\Omega} \xi_{n}(\mathbf{z}-\overline{\mathbf{z}}) T(u)\left(\nabla_{y} T_{\varepsilon}(u-\bar{u})^{+}+\nabla_{x} T_{\varepsilon}(u-\bar{u})^{+}\right) d x d y
\end{aligned}
$$




$$
\begin{aligned}
& +\int_{\Omega} \int_{\Omega} \xi_{n} \overline{\mathbf{z}}(T(u)-T(\bar{u}))\left(\nabla_{y} T_{\varepsilon}(u-\bar{u})^{+}+\nabla_{x} T_{\varepsilon}(u-\bar{u})^{+}\right) d x d y \\
= & : A^{1}+A^{2}
\end{aligned}
$$

Let us estimate $A^{1}$. First, observe that

$$
\begin{aligned}
\nabla_{y} T_{\varepsilon}(u-\bar{u}(x))^{+}(y) & =\chi_{(\bar{u}(x), \bar{u}(x)+\varepsilon)}(u(y)) \nabla_{y} u(y), \\
\nabla_{x} T_{\varepsilon}(u(y)-\bar{u})^{+}(x) & =-\chi_{(u(y)-\varepsilon, u(y))}(\bar{u}(x)) \nabla_{x} \bar{u}(x) \\
& =-\chi_{(\bar{u}(x), \bar{u}(x)+\varepsilon)}(u(y)) \nabla_{x} \bar{u}(x) .
\end{aligned}
$$

Since

$$
(\mathbf{a}(z, \xi)-\mathbf{a}(\hat{z}, \hat{\xi})) \cdot(\xi-\hat{\xi}) \geq-C|z-\hat{z}|\|\xi-\hat{\xi}\|
$$

for any $(z, \xi),(\hat{z}, \xi) \in \mathbb{R} \times \mathbb{R}^{N},|z|,|\hat{z}| \leq R$, we have

$$
\begin{aligned}
A^{1}= & \int_{\Omega} \int_{\Omega} \xi_{n}(\mathbf{z}-\overline{\mathbf{z}}) T(u)\left(\nabla_{y} u-\nabla_{x} \bar{u}\right) \chi_{(\bar{u}(x), \bar{u}(x)+\varepsilon)}(u) d x d y \\
\geq & -C\|T(u)\|_{\infty} \int_{\Omega} \int_{\Omega} \chi_{[u>a]} \xi_{n} \chi_{(\bar{u}(x), \bar{u}(x)+\varepsilon)}(u)|u-\bar{u}|\left\|\nabla_{y} u-\nabla_{x} \bar{u}\right\| d x d y \\
\geq & -\tilde{C} \int_{\Omega^{2}} \xi_{n} \chi_{\left(T_{\frac{a}{2}}^{\infty} \bar{u}(x), T_{\frac{a}{2}}^{\infty} \bar{u}(x)+\varepsilon\right)}\left(T_{a}^{\infty} u\right)\left|T_{a}^{\infty} u-T_{\frac{a}{2}}^{\infty} \bar{u}\right| \| \nabla_{y} T_{a}^{\infty} u \\
& -\nabla_{x} T_{\frac{a}{2}}^{\infty} \bar{u} \| d x d y .
\end{aligned}
$$

Having in mind (3) and applying Lemma 3 we obtain that

$$
\begin{aligned}
\lim _{n \rightarrow \infty} A^{1} & \geq-\tilde{C} \int_{\Omega} \psi(y) \chi_{\left\{0<T_{a}^{\infty} u-T_{\frac{a}{2}}^{\infty} \bar{u}<\varepsilon\right\}}\left(T_{a}^{\infty} u-T_{\frac{a}{2}}^{\infty} \bar{u}\right)\left|\nabla T_{a}^{\infty} u-\nabla T_{\frac{a}{2}}^{\infty} \bar{u}\right| d y \\
& \geq-\tilde{C} \varepsilon \int_{\Omega} \psi(y) \chi_{\left\{0<T_{a}^{\infty} u-T_{\frac{a}{2}}^{\infty} \bar{u}<\varepsilon\right\}}\left|\nabla T_{a}^{\infty} u-\nabla T_{\frac{a}{2}}^{\infty} \bar{u}\right| d y \geq-\varepsilon o(\varepsilon)
\end{aligned}
$$

where $o(\varepsilon) \rightarrow 0$ when $\varepsilon \rightarrow 0^{+}$, and we have used coarea formula in the last inequality. Similarly,

$$
\begin{aligned}
\left|A^{2}\right| & =\left|\int_{\Omega} \int_{\Omega} \xi_{n} \overline{\mathbf{z}}(T(u)-T(\bar{u}))\left(\nabla_{y} u-\nabla_{x} \bar{u}\right) \chi_{[\bar{u}(x), \bar{u}(x)+\varepsilon]}(u) d x d y\right| \\
& \leq \int_{\Omega} \int_{\Omega} \chi_{[u \geq a-\varepsilon]} \chi[\bar{u} \geq a-\varepsilon] \chi[0 \leq u-\bar{u} \leq \varepsilon] \xi_{n}|u-\bar{u}|\left\|\nabla_{y} u-\nabla_{x} \bar{u}\right\| d x d y \leq \varepsilon o(\varepsilon) .
\end{aligned}
$$

Hence,

$$
\lim _{n \rightarrow+\infty} I_{2}^{2}(a c) \geq-\varepsilon o(\varepsilon) .
$$

Finally, let us compute $I_{2}^{2}(s)$. 


$$
\begin{aligned}
I_{2}^{2}(s)= & \int_{\Omega}\left(\int_{\Omega} \xi_{n}\left(\mathbf{z}, D_{y} J_{T S_{\varepsilon}^{\prime}, \bar{u}(x)}(u)\right)^{s}\right) d x \\
& -\int_{\Omega}\left(\int_{\Omega} \xi_{n} T(\bar{u}) \overline{\mathbf{z}} \cdot D_{y}^{s} T_{\varepsilon}(u-\bar{u})^{+}\right) d x \\
& +\int_{\Omega}\left(\int_{\Omega} \xi_{n}\left(\overline{\mathbf{z}}, D_{x} J_{T S_{\varepsilon}^{u(y) \prime}}(\bar{u})\right)^{s}\right) d y \\
& +\int_{\Omega}\left(\int_{\Omega} \xi_{n} T(u) \mathbf{z} \cdot D_{x}^{s} T_{\varepsilon}(u-\bar{u})^{+}\right) d y \\
\geq & \int_{\Omega}\left(\int_{\Omega} \xi_{n}\left(\left(h_{T}\left(u, D_{y} T_{\bar{u}}^{\bar{u}+\varepsilon}(u)\right)\right)^{s}-T(\bar{u}) \overline{\mathbf{z}} \cdot D_{y}^{s} T_{\varepsilon}(u-\bar{u})^{+}\right)\right) d x \\
& +\int_{\Omega}\left(\int_{\Omega} \xi_{n}\left(\left(h_{T}\left(\bar{u}, D_{x} T_{u-\varepsilon}^{u}(\bar{u})\right)\right)^{s}+T(u) \mathbf{z} \cdot D_{x}^{s} T_{\varepsilon}(u-\bar{u})^{+}\right)\right) d y \\
= & \int_{\Omega}\left(\int_{\Omega} \xi_{n}\left(\left|D_{y}^{s} J_{T \varphi}\left(T_{\bar{u}}^{\bar{u}+\varepsilon}(u)\right)\right|-T(\bar{u}) \overline{\mathbf{z}} \cdot D_{y}^{s} T_{\varepsilon}(u-\bar{u})^{+}\right)\right) d x \\
& +\int_{\Omega}\left(\int_{\Omega} \xi_{n}\left(\left|D_{x}^{s} J_{T \varphi}\left(T_{u-\varepsilon}^{u}(\bar{u})\right)\right|+T(u) \mathbf{z} \cdot D_{x}^{s} T_{\varepsilon}(u-\bar{u})^{+}\right)\right) d y \\
= & I_{2}^{2}(s, c)+I_{2}^{2}(s, j),
\end{aligned}
$$

where $\varphi(s)=s, I_{2}^{2}(s, c)$ collects all the Cantor parts of the measures and $I_{2}^{2}(s, j)$ their jump parts. For the Cantor part,

$$
\begin{aligned}
I_{2}^{2}(s, c)= & \int_{\Omega}\left(\int_{\Omega} \xi_{n} \chi_{\{0<u-\bar{u}<\varepsilon\}}\left(u T(u)\left|D^{c} u\right|-\bar{u} T(\bar{u}) \bar{z}_{b} \cdot D^{c} u\right)\right) d x \\
& +\int_{\Omega}\left(\int_{\Omega} \xi_{n} \chi_{\{0<u-\bar{u}<\varepsilon\}}\left(\bar{u} T(\bar{u})\left|D^{c} \bar{u}\right|-u T(u) z_{b} \cdot D^{c} \bar{u}\right)\right) d y \\
\geq & \int_{\Omega}\left(\int_{\Omega} \xi_{n} \chi_{\{0<u-\bar{u}<\varepsilon\}}(u T(u)-\bar{u} T(\bar{u}))\left|D^{c} u\right|\right) d x \\
& +\int_{\Omega}\left(\int_{\Omega} \xi_{n} \chi_{\{0<u-\bar{u}<\varepsilon\}}(\bar{u} T(\bar{u})-u T(u))\left|D^{c} \bar{u}\right|\right) d y \\
= & \int_{\Omega}\left(\int_{\Omega} \xi_{n} \chi_{\{0<u-\bar{u}<\varepsilon\}}(u T(u)-\bar{u} T(\bar{u})) d x\right) d\left|D^{c} u\right| \\
& +\int_{\Omega}\left(\int_{\Omega} \xi_{n} \chi_{\{0<u-\bar{u}<\varepsilon\}}(\bar{u} T(\bar{u})-u T(u)) d y\right) d\left|D^{c} \bar{u}\right| \\
= & \int_{\Omega \cap\{u>a\}}\left(\int_{\Omega} \xi_{n} \chi_{\{0<u-\bar{u}<\varepsilon\} \cap\{\bar{u}>a-\varepsilon\}}(u T(u)-\bar{u} T(\bar{u})) d x\right) d\left|D^{c} u\right| \\
& +\int_{\Omega \cap\{\bar{u}>a-\varepsilon\}}\left(\int_{\Omega} \xi_{n} \chi_{\{0<u-\bar{u}<\varepsilon\} \cap\{u>a\}}(\bar{u} T(\bar{u})-u T(u)) d y\right) d\left|D^{c} \bar{u}\right| .
\end{aligned}
$$

By Lemma 2, taking $F(s)=s T(s), w=T_{a}^{\infty}(u)$ and $\bar{w}=T_{a-\varepsilon}^{\infty}(\bar{u})$ and having in mind (4), we have that 


$$
\begin{aligned}
& \lim _{n \rightarrow+\infty} I_{2}^{2}(s, c) \\
& \geq \int_{\Omega} \psi \chi_{\{0<u-\bar{u}<\varepsilon\} \cap\{u>a\} \cap\{\bar{u}>a-\varepsilon\}}(u T(u)-\bar{u} T(\bar{u})) d\left(\left|D^{c} u\right|-\left|D^{c} \bar{u}\right|\right) \\
& \geq-C \varepsilon \int_{\Omega} \chi_{\left\{0<T_{a}^{\infty} u-T_{a-\varepsilon}^{\infty} \bar{u}<\varepsilon\right\}} d\left|D^{c}\left(T_{a}^{\infty}(u)-T_{a-\varepsilon}^{\infty}(\bar{u})\right)\right| \geq-\varepsilon o(\varepsilon) .
\end{aligned}
$$

For the jump part,

$$
\begin{aligned}
I_{2}^{2}(s, j) & \geq \int_{\Omega}\left(\int_{\Omega} \xi_{n}\left(\left|D_{y}^{j} J_{T \varphi}\left(T_{\bar{u}}^{\bar{u}+\varepsilon}(u)\right)\right|-\bar{u} T(\bar{u})\left|D_{y}^{j} T_{\bar{u}}^{\bar{u}+\varepsilon}(u)\right|\right)\right) d x \\
& +\int_{\Omega}\left(\int_{\Omega} \xi_{n}\left(\left|D_{x}^{j} J_{T \varphi}\left(T_{u-\varepsilon}^{u}(\bar{u})\right)\right|-u T(u)\left|D_{x}^{j} T_{u-\varepsilon}^{u}(\bar{u})\right|\right)\right) d y
\end{aligned}
$$

Observe now that

$$
\begin{aligned}
& \left|D_{y}^{j} J_{T \varphi}\left(T_{\bar{u}}^{\bar{u}+\varepsilon}(u)\right)\right|-\bar{u} T(\bar{u})\left|D_{y}^{j} T_{\bar{u}}^{\bar{u}+\varepsilon}(u)\right| \\
& \quad=\left(\int_{T_{\bar{u}}^{\bar{u}+\varepsilon}\left(u^{-}\right)}^{T_{\bar{u}+\varepsilon}^{\bar{u}}\left(u^{+}\right)} T(s) s d s-\bar{u} T(\bar{u})\left(T_{\bar{u}}^{\bar{u}+\varepsilon}\left(u^{+}\right)-T_{\bar{u}}^{\bar{u}+\varepsilon}\left(u^{-}\right)\right)\right) \mathcal{H}^{N-1}\left\llcorner_{J_{\bar{u}}^{\bar{u}+\varepsilon}(u)}\right. \\
& \quad=\left(\left(\xi_{u^{ \pm}, \bar{u}, \varepsilon} T\left(\xi_{u^{ \pm}, \bar{u}, \varepsilon}\right)-\bar{u} T(\bar{u})\right)\left(T_{\bar{u}}^{\bar{u}+\varepsilon}\left(u^{+}\right)-T_{\bar{u}}^{\bar{u}+\varepsilon}\left(u^{-}\right)\right)\right) \mathcal{H}^{N-1} L_{J_{\bar{u}}^{\bar{u}+\varepsilon}(u)} \geq 0,
\end{aligned}
$$

with $\xi_{u^{ \pm}, \bar{u}, \varepsilon} \in\left[T_{\bar{u}}^{\bar{u}+\varepsilon}\left(u^{-}\right), T_{\bar{u}}^{\bar{u}+\varepsilon}\left(u^{+}\right)\right]$. Similarly, as measures,

$$
\begin{aligned}
& \left|D_{x}^{j} J_{T \varphi}\left(T_{u-\varepsilon}^{u}(\bar{u})\right)\right|-u T(u)\left|D_{x}^{j} T_{u-\varepsilon}^{u}(\bar{u})\right| \\
& \quad=\left(\left(\xi_{u^{ \pm}, \bar{u}, \varepsilon} T\left(\xi_{\bar{u}^{ \pm}, u, \varepsilon}\right)-u T(u)\right)\left(T_{u-\varepsilon}^{u}\left(\bar{u}^{+}\right)-T_{u-\varepsilon}^{u}\left(\bar{u}^{-}\right)\right)\right) \mathcal{H}^{N-1}\left\llcorner_{T_{u-\varepsilon}^{u}(\bar{u})}\right. \\
& \quad \geq-C \varepsilon^{2} \mathcal{H}^{N-1}\left\llcorner_{T_{a-\varepsilon} \infty}(\bar{u}) .\right.
\end{aligned}
$$

Therefore,

$$
I_{2}^{2}(s, j) \geq-C \varepsilon^{2} \int_{\Omega} \int_{J_{T_{a-\varepsilon}^{\infty}(\bar{u})}} \xi_{n} d \mathcal{H}^{N-1} d y \geq-C \varepsilon^{2} \int_{J_{T_{a-\varepsilon}^{\infty}(\bar{u})}} d \mathcal{H}^{N-1} \geq-C \varepsilon^{2} .
$$

Collecting all these facts, we obtain

$$
\lim _{n \rightarrow \infty} I_{2}^{2} \geq \varepsilon o(\varepsilon)
$$

Letting $n \rightarrow \infty$ in (5), we have

$$
\begin{aligned}
& \int_{\Omega} \psi(u T(u)-\bar{u} T(\bar{u})) T_{\varepsilon}(u-\bar{u})^{+} d x \\
& \quad+\int_{\Omega} T_{\varepsilon}(u-\bar{u})^{+}(T(u) \mathbf{z}-T(\bar{u}) \overline{\mathbf{z}}) \cdot \nabla \psi(x) d x \\
& \quad \leq \int_{\Omega} \psi(v T(u)-\bar{v} T(\bar{u})) T_{\varepsilon}(u-\bar{u})^{+} d x-\varepsilon \int_{\Omega} \psi(\overline{\mathbf{z}}, D T(\bar{u}))+\varepsilon o(\varepsilon) .
\end{aligned}
$$


We take now a sequence $\psi_{m} \uparrow \chi_{\Omega}, \psi_{m} \in \mathcal{D}(\Omega)$ in the above formula. Then,

$$
\begin{aligned}
& \int_{\Omega}(u T(u)-\bar{u} T(\bar{u})) T_{\varepsilon}(u-\bar{u})^{+} d x \\
& \quad+\limsup _{m \rightarrow \infty} \int_{\Omega} T_{\varepsilon}(u-\bar{u})^{+}(T(u) \mathbf{z}-T(\bar{u}) \overline{\mathbf{z}}) \cdot \nabla \psi_{m} d x \\
& \quad \leq \int_{\Omega}(v T(u)-\bar{v} T(\bar{u})) T_{\varepsilon}(u-\bar{u})^{+} d x-\varepsilon \int_{\Omega}(\overline{\mathbf{z}}, D T(\bar{u}))+\varepsilon O(\varepsilon) .
\end{aligned}
$$

Let us see that the second term in the above expression is nonnegative.

$$
\begin{aligned}
\int_{\Omega} & T_{\varepsilon}(u-\bar{u})^{+}(T(u) \mathbf{z}-T(\bar{u}) \overline{\mathbf{z}}) \cdot \nabla \psi_{m}(x) d x \\
= & -\int_{\Omega} \psi_{m} T_{\varepsilon}(u-\bar{u})^{+} T(u) \operatorname{div}(\mathbf{z}) d x-\int_{\Omega} \psi_{m}\left(\mathbf{z}, D\left(T_{\varepsilon}(u-\bar{u})^{+} T(u)\right)\right) \\
& +\int_{\Omega} \psi_{m} T_{\varepsilon}(u-\bar{u})^{+} T(\bar{u}) \operatorname{div}(\overline{\mathbf{z}}) d x+\int_{\Omega} \psi_{m}\left(\overline{\mathbf{z}}, D\left(T_{\varepsilon}(u-\bar{u})^{+} T(\bar{u})\right)\right) .
\end{aligned}
$$

Now, since $T_{\varepsilon}(u-\bar{u}) T(u) \in B V(\Omega), u \geq g, \bar{u} \geq g$ in $\partial \Omega$,

$$
\{x \in \partial \Omega: u(x)>\bar{u}(x)\} \subset\{x \in \partial \Omega: u(x)>g(x)\},
$$

$[\mathbf{z}, v]=u \kappa$ with $\kappa \in \operatorname{sign}(g-u)$ in $\partial \Omega \cap\{u>0\}$, and $[\overline{\mathbf{z}}, v]=\bar{u} \bar{\kappa}$ with $\bar{\kappa} \in$ $\operatorname{sign}(g-\bar{u})$ in $\partial \Omega \cap\{\bar{u}>0\}$, we have

$$
\begin{aligned}
& \lim _{m \rightarrow \infty} \int_{\Omega} T_{\varepsilon}(u-\bar{u})^{+}(T(u) \mathbf{z}-T(\bar{u}) \overline{\mathbf{z}}) \cdot \nabla \psi_{m}(x) d x \\
& =-\int_{\Omega} T_{\varepsilon}(u-\bar{u})^{+} T(u) \operatorname{div}(\mathbf{z}) d x-\int_{\Omega}\left(\mathbf{z}, D\left(T_{\varepsilon}(u-\bar{u})^{+} T(u)\right)\right) \\
& +\int_{\Omega} T_{\varepsilon}(u-\bar{u})^{+} T(\bar{u}) \operatorname{div}(\overline{\mathbf{z}}) d x+\int_{\Omega}\left(\overline{\mathbf{z}}, D\left(T_{\varepsilon}(u-\bar{u})^{+} T(\bar{u})\right)\right) \\
& =-\int_{\partial \Omega}([\mathbf{z}, v] T(u)-[\overline{\mathbf{z}}, \nu] T(\bar{u})) T_{\varepsilon}(u-\bar{u})^{+} d \mathcal{H}^{N-1} \\
& =\int_{[u>\bar{u}] \cap \partial \Omega}\left([\overline{\mathbf{z}}, v] \chi_{\{\bar{u}>a\}} T(\bar{u})-[\mathbf{z}, \nu] \chi_{\{u>a\}} T(u)\right) T_{\varepsilon}(u-\bar{u})^{+} d \mathcal{H}^{N-1} \\
& =\int_{[u>\bar{u}] \cap \partial \Omega}(\bar{u} \bar{\kappa} T(\bar{u})-u \kappa T(u)) T_{\varepsilon}(u-\bar{u})^{+} d \mathcal{H}^{N-1} \\
& \geq \int_{[u>\bar{u}] \cap \partial \Omega}(u T(u)-\bar{u} T(\bar{u})) T_{\varepsilon}(u-\bar{u})^{+} d \mathcal{H}^{N-1} \geq 0 .
\end{aligned}
$$

Therefore,

$$
\int_{\Omega}(u T(u)-\bar{u} T(\bar{u})) T_{\varepsilon}(u-\bar{u})^{+} d x
$$




$$
\leq \int_{\Omega}(v T(u)-\bar{v} T(\bar{u})) T_{\varepsilon}(u-\bar{u})^{+} d x-\varepsilon \int_{\Omega}(\overline{\mathbf{z}}, D T(\bar{u}))+\varepsilon o(\varepsilon) .
$$

Dividing the last expression by $\varepsilon$ and letting $\varepsilon \rightarrow 0$, letting $a \rightarrow 0^{+}$, and finally dividing by $b$ and letting $b \rightarrow 0^{+}$we obtain (42) in the original article. As explained before, the rest of the proof is already written in the 150-151 pages of the original article.

Sketch of proof of uniqueness of Theorem 3 of the original article: First of all, we note that given $a>0$, by Lemma 1 there exist $\varepsilon \rightarrow 0$ such that

$$
\mathcal{L}^{N+2}\left(\left\{(x, s, t): T_{a}^{\infty}\left(u(s, x)-T_{\frac{a}{2}}^{\infty} \bar{u}(t, x)\right)=\varepsilon\right\}\right)=0
$$

and

$$
\int_{(0, T)^{2}}\left(\left|D^{c} T_{a}^{\infty} u(t)\right|+\left|D^{c} T_{\frac{a}{2}}^{\infty} \bar{u}(s)\right|\right)\left(\left\{\widehat{T_{a}^{\infty} u(s)}-\widetilde{T_{\frac{a}{2}}^{\infty} \bar{u}(t)}=\varepsilon\right\}\right) d s d t=0 .
$$

As stated before, we only sketch here how to pass from (124) to (130) in the original article. (124) reads as

$$
\begin{aligned}
& -\int_{\left(Q_{T}\right)^{2}}\left(J_{T, \varepsilon, \bar{u}}^{+}(u)\left(\eta_{m, n}\right)_{t}+J_{T, \varepsilon, u}^{-}(\bar{u})\left(\eta_{m, n}\right)_{s}\right)-\varepsilon \int_{\left(Q_{T}\right)^{2}} J_{T}(\bar{u})\left(\eta_{m, n}\right)_{s} d y d s \\
& +\int_{\left(Q_{T}\right)^{2}} \eta_{m, n} h_{T}\left(u, D_{x} S_{\varepsilon, \bar{u}}(u)\right)+\int_{\left(Q_{T}\right)^{2}} \eta_{m, n} h_{T}\left(\bar{u}, D_{y} S_{\varepsilon}^{u}(\bar{u})\right) \\
& -\int_{\left(Q_{T}\right)^{2}} \overline{\mathbf{z}} \cdot \nabla_{x} \eta_{m, n} T(\bar{u}) S_{\varepsilon}^{u}(\bar{u})-\int_{\left(Q_{T}\right)^{2}} \mathbf{z} \cdot \nabla_{y} \eta_{m, n} T(u) S_{\varepsilon, \bar{u}}(u) \\
& +\int_{\left(Q_{T}\right)^{2}} T_{\varepsilon}(u-\bar{u})^{+}(T(u) \mathbf{z}-T(\bar{u}) \overline{\mathbf{z}}) \cdot\left(\nabla_{x} \eta_{m, n}+\nabla_{y} \eta_{m, n}\right) \\
& +\varepsilon \int_{\left(Q_{T}\right)^{2}} T(\bar{u}) \overline{\mathbf{z}} \cdot\left(\nabla_{x} \eta_{m, n}+\nabla_{y} \eta_{m, n}\right) \leq 0,
\end{aligned}
$$

with $\eta_{m, n}(t, x, s, y):=\rho_{m}(x-y) \tilde{\rho}_{n}(t-s) \phi\left(\frac{t+s}{2}\right) \psi\left(\frac{x+y}{2}\right)$, being $\rho_{m}$ a sequence of mollifiers in $\Omega$ and $\tilde{\rho}_{n}$ a sequence of mollifiers in $\mathbb{R}$. Let $I_{1}, I_{2}$ be, respectively, the sum of the first two terms and the sum of the third up to the sixth terms of the above inequality. Working as in the proof of Theorem 1, (using Lemmas 4 and 5) we get

$$
\lim _{m \rightarrow+\infty} I_{2} \geq \varepsilon o(\varepsilon)-\varepsilon \int_{(0, T)^{2} \times \Omega} T(\bar{u}) \overline{\mathbf{z}} \cdot \nabla \chi_{n} .
$$

Hence, by (8), it follows that

$$
-\int_{(0, T)^{2} \times \Omega}\left(J_{T, \varepsilon, \bar{u}}^{+}(u)\left(\chi_{n}\right)_{t}+J_{T, \varepsilon, u}^{-}(\bar{u})\left(\chi_{n}\right)_{s}\right)
$$




$$
\begin{aligned}
& +\int_{(0, T)^{2} \times \Omega} T_{\varepsilon}(u-\bar{u})^{+}(T(u) \mathbf{z}-T(\bar{u}) \overline{\mathbf{z}}) \cdot \nabla \chi_{n}-\varepsilon \int_{(0, T)^{2} \times \Omega} T(\bar{u}) \overline{\mathbf{z}} \cdot \nabla \chi_{n} \\
\leq & \varepsilon O(\varepsilon)+\varepsilon \int_{(0, T)^{2} \times \Omega} J_{T}(\bar{u})\left(\chi_{n}\right)_{s},
\end{aligned}
$$

where $\chi_{n}(t, s, x):=\tilde{\rho}_{n}(t-s) \phi\left(\frac{t+s}{2}\right) \psi(x)$. Letting now $\psi=\psi_{m} \uparrow \chi_{\Omega}$ we get,

$$
\begin{aligned}
& -\int_{(0, T)^{2} \times \Omega}\left(J_{T, \varepsilon, \bar{u}}^{+}(u)\left(\kappa_{n}\right)_{t}+J_{T, \varepsilon, u}^{-}(\bar{u})\left(\kappa_{n}\right)_{s}\right) \\
& +\liminf _{m \rightarrow+\infty} \int_{(0, T)^{2} \times \Omega} \kappa_{n} T_{\varepsilon}(u-\bar{u})^{+}(T(u) \mathbf{z}-T(\bar{u}) \overline{\mathbf{z}}) \cdot \nabla \psi_{m} \\
& -\varepsilon \liminf _{m \rightarrow+\infty} \int_{(0, T)^{2} \times \Omega} \kappa_{n} T(\bar{u}(s, x)) \overline{\mathbf{z}}(s, x) \cdot \nabla \psi_{m} \leq \varepsilon \int_{(0, T)^{2} \times \Omega} J_{T}(\bar{u}(s, x))\left(\kappa_{n}\right)_{s},
\end{aligned}
$$

where $\kappa_{n}(t, s):=\tilde{\rho}_{n}(t-s) \phi\left(\frac{t+s}{2}\right)$. Using now Steklov's type averages, it is possible to prove the following

\section{Claim}

$$
\liminf _{m \rightarrow+\infty} \int_{(0, T)^{2} \times \Omega} \kappa_{n} T(\bar{u}) \overline{\mathbf{z}} \cdot \nabla \psi_{m}=-\int_{0}^{T} \int_{0}^{T} \int_{\partial \Omega}[\overline{\mathbf{z}}, \nu] \kappa_{n} T(\bar{u}) d \mathcal{H}^{N-1} d t d s,
$$

and

$$
\begin{aligned}
& \liminf _{m \rightarrow+\infty} \int_{(0, T)^{2} \times \Omega} \kappa_{n} T_{\varepsilon}(u-\bar{u})^{+}(T(u) \mathbf{z}-T(\bar{u}) \overline{\mathbf{z}}) \cdot \nabla \psi_{m} \\
& \quad=-\int_{(0, T)^{2}} \int_{\partial \Omega} \kappa_{n} T_{\varepsilon}(u-\bar{u})^{+}([\mathbf{z}, v] T(u)-[\overline{\mathbf{z}}, v] T(\bar{u})) d \mathcal{H}^{N-1}
\end{aligned}
$$

Once the claim is proved, by (10) and (11), dividing (9) by $\varepsilon$ and letting $\varepsilon \rightarrow 0^{+}$ we obtain (130) in the original article and the proof finishes. To finish this note we give the proof of (10) for the sake of completeness (the proof of (11) is analogous).

Proof of the claim For $\tau>0$, we define the function $\left[\kappa_{n}(s)\right]^{\tau}$, as the Dunford integral

$$
\left[\kappa_{n}(s)\right]^{\tau}(t)=\frac{1}{\tau} \int_{t}^{t+\tau} \kappa_{n}(r, s) T(\bar{u}(r, x)) d r
$$

Then, since $\bar{\xi}=\operatorname{div}(\overline{\mathbf{z}})$ in the sense of Definition 4 in the original article, we have

$$
I_{m}:=\int_{(0, T)^{2} \times \Omega} \kappa_{n} T(\bar{u}(s, x)) \overline{\mathbf{z}}(s, x) \cdot \nabla \psi_{m}
$$




$$
\begin{aligned}
= & \lim _{\tau \rightarrow 0} \int_{(0, T)^{2} \times \Omega}\left[\kappa_{n}(s)\right]^{\tau}(t) \overline{\mathbf{z}}(t, x) \cdot \nabla\left(\psi_{m}(x)-1\right) \\
= & -\lim _{\tau \rightarrow 0}\left\{\int_{0}^{T} \int_{Q_{T}}\left(\psi_{m}(x)-1\right)\left(\overline{\mathbf{z}}, D_{x}\left(\left[\kappa_{n}(s)\right]^{\tau}\right)\right) d s\right. \\
& \left.+\int_{0}^{T} \int_{0}^{T}\left\langle\bar{\xi}(t),\left[\kappa_{n}(s)\right]^{\tau}(t)\left(\psi_{m}-1\right)\right\rangle d t d s\right\} \\
& +\lim _{\tau \rightarrow 0} \int_{0}^{T} \int_{0}^{T} \int_{\partial \Omega}[\overline{\mathbf{z}}(t), v]\left(\psi_{m}-1\right)\left[\kappa_{n}(s)\right]^{\tau}(t) d \mathcal{H}^{N-1} d t d s=: I_{m}^{1}+I_{m}^{2}+I_{m}^{3} .
\end{aligned}
$$

Observe that limit in the l.h.s. above as $\tau \rightarrow 0^{+}$exists. We prove that the limit of $I_{m}^{3}$ exists, hence also the limit of $I_{m}^{1}+I_{m}^{2}$. It is not difficult to see (see the proof of (109) in the original article) that

$$
\left|D\left(\left[\kappa_{n}(s)\right]^{\tau}(t)\right)\right|(\Omega) \stackrel{\tau \rightarrow 0}{\longrightarrow}\left|D\left(\kappa_{n} T(\bar{u}(t))\right)\right|(\Omega) .
$$

Hence,

$$
I_{m}^{3}=-\int_{0}^{T} \int_{0}^{T} \int_{\partial \Omega}[\overline{\mathbf{z}}(t), \nu] \kappa_{n} T(\bar{u}(t)) d \mathcal{H}^{N-1} d t d s .
$$

On the other hand, by (12),

$$
\begin{aligned}
\left|I_{m}^{1}\right| & \leq \limsup _{\tau \rightarrow 0}\|\overline{\mathbf{z}}\|_{\infty} \int_{0}^{T} \int_{Q_{T}}\left(1-\psi_{m}\right)\left|D\left(\left[\kappa_{n}(s)\right]^{\tau}(t)\right)\right| \\
& =\|\overline{\mathbf{z}}\|_{\infty} \int_{0}^{T} \int_{Q_{T}}\left(1-\psi_{m}\right)\left|D\left(\kappa_{n} T(\bar{u}(t))\right)\right|
\end{aligned}
$$

which implies that $\lim _{m \rightarrow \infty} I_{m}^{1}=0$. Then,

$$
\begin{aligned}
I_{m}^{2} & =-\lim _{\tau \rightarrow 0} \int_{0}^{T} \int_{0}^{T}\left\langle\bar{\xi}(t),\left[\kappa_{n}(s)\right]^{\tau}(t)\left(\psi_{m}-1\right)\right\rangle d t d s \\
& =\lim _{\tau \rightarrow 0} \int_{0}^{T} \int_{0}^{T} \int_{\Omega} \bar{u}(t, x) \frac{T(\bar{u}(t+\tau)) \kappa_{n}(t+\tau)-T(\bar{u}(t)) \kappa_{n}(t)}{\tau}\left(\psi_{m}-1\right) .
\end{aligned}
$$

Let $Q(r):=\int_{0}^{r} T(\tau) d \tau$. Therefore, $Q(r)-Q(s) \leq T(r)(r-s)$. Thus,

$$
\begin{aligned}
I_{m}^{2} & =\lim _{\tau \rightarrow 0} \int_{0}^{T} \int_{0}^{T} \int_{\Omega}\left(1-\psi_{m}\right) \frac{\bar{u}(t)-\bar{u}(t-\tau)}{\tau} T(\bar{u}(t)) \kappa_{n}(t) \\
& \geq \lim _{\tau \rightarrow 0} \int_{0}^{T} \int_{0}^{T} \int_{\Omega}\left(1-\psi_{m}\right) \kappa_{n}(t, s) \frac{Q(\bar{u}(t))-Q(\bar{u}(t-\tau))}{\tau} \\
& =\lim _{\tau \rightarrow 0} \int_{0}^{T} \int_{0}^{T} \int_{\Omega}\left(1-\psi_{m}\right) Q(\bar{u}(t)) \frac{\kappa_{n}(t, s)-\kappa_{n}(t+\tau, s)}{\tau}
\end{aligned}
$$




$$
=-\int_{0}^{T} \int_{0}^{T} \int_{\Omega}\left(1-\psi_{m}\right) Q(\bar{u}(t)) \frac{d \kappa_{n}(t, s)}{d t} \stackrel{m \rightarrow+\infty}{\longrightarrow} 0 .
$$

Therefore,

$$
\liminf _{m \rightarrow \infty} I_{m}^{2} \geq 0
$$

Taking into account the above facts, we get

$$
\liminf _{m \rightarrow \infty} I_{m} \geq-\int_{0}^{T} \int_{0}^{T} \int_{\partial \Omega}[\mathbf{z}(t), v] \kappa_{n} T(\bar{u}(t)) d \mathcal{H}^{N-1} d t d s
$$

In order to obtain the opposite inequality, we work similarly with

$$
\left[\kappa_{n}(s)\right]_{\tau}(t):=\frac{1}{\tau} \int_{t-\tau}^{t} \kappa_{n}(r, s) T(\bar{u}(r, x)) d r
$$

and the claim is proved.

Acknowledgments The authors have been partially supported by the Spanish MEC project, reference MTM2012 -31103. The authors would like to acknowledge L. Giacomelli and F. Petitta for several and fruitful discussions.

\section{Reference}

1. Ambrosio, L., Fusco, N., Pallara, D.: Functions of bounded variation and free discontinuity problems. Oxford Mathematical Monographs, Oxford (2000) 